\title{
Effect of willed movement training on neurorehabilitation after focal cerebral ischemia and on the neural plasticity-associated signaling pathway
}

\author{
ZHI-WEN ZHOU ${ }^{1}$, QI-DONG YANG ${ }^{1}$, QING-PING TANG ${ }^{2,3}$, JIE YANG ${ }^{1}$, RONG-JING GUO ${ }^{1}$ and WEN JIANG ${ }^{1}$ \\ ${ }^{1}$ Department of Neurology, Xiangya Hospital, Central South University; ${ }^{2}$ Department of Physiology, School of \\ Basic Medical Sciences, Central South University, Changsha, Hunan 410008; ${ }^{3}$ Department of Rehabilitation, \\ Brain Hospital of Hunan Province, Hunan University of Chinese Medicine, Changsha, Hunan 410007, P.R. China
}

Received June 3, 2017; Accepted September 12, 2017

DOI: $10.3892 / \mathrm{mmr} .2017 .7964$

\begin{abstract}
Neurorehabilitation training is a therapeutic intervention for the loss of neural function induced by focal cerebral ischemia, however, the effect varies depending on the neurorehabilitation exercises. Willed movement (WM) training is defined as task-oriented training, which increases enthusiasm of patients to accomplish a specific task. The current study was performed to the evaluate effect of WM training on neurorehabilitation following focal cerebral ischemia, and further investigate the influence on neural plasticity-associated signaling pathway. Sprague-Dawley rats following temporary middle cerebral artery occlusion (tMCAO) were randomly divided into four groups: tMCAO (no rehabilitation training), CR (control rehabilitation), EM (environmental modification) and WM groups. Rats in the CR group were forced to exercise (running) in a rotating wheel. In the WM group, food was used to entice rats to climb on a herringbone ladder. Herringbone ladders were also put into the cages of the rats in the CR and EM groups, however without the food attraction. WM group exhibited an improvement in neurobehavioral performance compared with other groups. TTC staining indicated an evident reduction in brain damage in the WM group. There were increased synaptic junctions following WM training, based on the observations of transmission election microscopy. Investigation of the molecular mechanism suggested that WM training conferred the greatest effect on stimulating
\end{abstract}

Correspondence to: Dr Zhi-Wen Zhou, Department of Neurology, Xiangya Hospital, Central South University, 87 Xiang Ya Road, Changsha, Hunan 410008, P.R. China

E-mail: 15409979@qq.com

Dr Qing-Ping Tang, Department of Physiology, School of Basic Medical Sciences, Central South University, 90 Xiang Ya Road, Changsha, Hunan 410008, P.R. China

E-mail: tqingping1111@126.com

Key words: willed movement training, focal cerebral ischemia, ERK/CREB pathway, GluR2/GRASP-1/PICK1 cascades the extracellular signal-related kinase (ERK)/cyclic adenosine monophosphate response element-binding protein 1 (CREB) pathway and glutamate receptor 2 (GluR2)/glutamate receptor interacting protein 1-associated protein 1 (GRASP-1)/protein interacting with $\mathrm{C}$-kinase 1 (PICK1) cascades among groups. Collectively, the improvement of neurobehavioral performance by WM training following tMCAO is suggested to involve the ERK/CREB pathway and GluR2/GRASP-1/PICK1 cascades. The present study provided a preliminary foundation for future research on the therapeutic effect of WM training against stroke-induced neuron damage.

\section{Introduction}

Ischemic stroke is a threat to human health with the third leading cause of adult disability and mortality worldwide (1). As the most common type of stroke, cerebral focal ischemia is associated with neuronal apoptosis or mortality, glial cell activation and proliferation, inflammatory reaction and stress response, resulting in a series of nonreversible neuronal loss and neurological deficits (2). Due to the fact that the central nervous system of adults has limited self-repair and regeneration abilities, survivors are likely to sustain lifelong impairments in behavioral, communicative, cognitive, sensory and/or emotional functionality, depending on the size and localization of the brain injury (2). Although drug therapies may prevent the loss of nerve cells after cerebral focal ischemia, the therapeutic effect on restoring neural function in patients remains limited (3).

In this context, neurorehabilitation exercise has been developed to help stroke patients improve the impairments to the nervous system and motor control, according to the neural plasticity. Neuroplasticity refers to a range of adaptive changes that occur in the structure and function of cells in the nervous system in response to physiological or pathological perturbations (4). These changes include the sprouting and growth of axons or dendrites, synapse formation, the strengthening of synapses in response to repeated activation and the production of new neurons derived from differentiation of stem cells $(4,5)$. Motor training is able to enhance the neuronal structural and functional synaptic plasticity in the 
motor cortex; however, the effect varies depending on the neurorehabilitation exercises used. Willed movement (WM) training is defined as task-oriented training, which increases the enthusiasm of patients to accomplish a specific task (6). In this training, patients are actively participating in physical activities leading to profound changes in neuroplasticity, which is likely to be associated to a better therapeutic effect on the neurorehabilitation (6).

The extracellular signal-related kinase (ERK)-mediated pathway is involved in neural plasticity and neuroprotection following stroke. Endogenous neural regeneration in the hippocampus represents a special type of neural plasticity. Neural stem cells, located in the subgranular zone of hippocampal dentate gyrus, are capable of proliferating, differentiating and integrating into the existing neuronal circuits. These self-renewing cells have the potential to replenish neural loss and restore neurological function after stroke (7). Post-traumatic neurogenesis and cognition recovery has been previously observed to be rescued by the activation of mitogen-activated protein kinase (MEK)/ERK (7). Conditional activation of ERK enhances hippocampus neurogenesis in a rat model of traumatic brain injury, improving the olfactory function under normal conditions and after injury, and inhibition of MEK/ERK abolished the neurogenesis improved by sphingosine-1-phosphate receptor 1 activation (7). The pivotal role of ERK in endogenous neurogenesis is likely mediated through regulating multiple factors, including cyclic adenosine monophosphate response element-binding protein (CREB), brain-derived neurotrophic factor (BDNF), nerve growth factor, and synaptophysin (SYP) $(8,9)$. Notably, it has been previously suggested that there is a critical role of CREB activation in plasticity of dendritic spines in central neurons $(8,9)$. The CREB-mediated pathway is implicated in enhanced structural plasticity and the behavioral recovery that are promoted by post-stroke forced limb-use in cerebral multi-infarction rats (10). In addition, resveratrol-mediated neuroprotection in a chronic cerebral hypoperfusion rat model has been suggested to act via CREB activation that restores functional and structural synaptic plasticity (11). The neuroprotection of CREB activation may also be associated with the effect on DNA repair and anti-oxidation (12). It is known that reactive oxygen species resulting from cerebral ischemia and reperfusion lead to significant neuronal damage (12).

The critical role of glutamate receptor 2 (GluR2) in activity-dependent forms of synaptic plasticity has been previously reported (13). GluR2, known to be one of the subunits of $\alpha$-amino-3-hydroxy-5-methyl-4-isoxazolepropi onic acid receptors (AMPARs), negatively regulates calcium permeability, resulting in a marked inward rectification in the current-voltage relationship (14). It has been previously reported that ischemia induces a delayed downregulation of GluR2 mRNA and protein expression, which enhances AMPAR-mediated $\mathrm{Ca}^{2+}$ and $\mathrm{Zn}^{2+}$ influx into CA1 neurons and subsequently activates the death pathway (14). This evidence indicates a crucial role for $\mathrm{Ca}^{2+}$-permeable AMPARs via loss of GluR2 in ischemic cell death. In addition, endocytosis and exocytosis mediated by AMPAR are central to the necessary changes in the synaptic receptor complement pathway (15). Protein interacting with C-kinase 1 (PICK1) is a calcium-sensing, PDZ domain-containing protein that interacts with GluR2 AMPAR subunit and regulates AMPAR trafficking. PICK1 over expression in CA1 pyramidal neurons causes a CaMK- and PKC-dependent potentiation of AMPAR-mediated transmission (14). Glutamate receptor interacting protein (GRIP) 1-associated protein 1 (GRASP-1) is a GRIP-associated protein that affects GluR2/3-GRIP interaction and regulates AMPAR trafficking to the synaptic membrane (16-18). Increased delivery of AMPARs to the postsynaptic membrane leads to long-term potentiation (LTP) and mice exhibit abnormal excitatory synapse number, synaptic plasticity, and hippocampal-dependent learning and memory due to a failure in learning-induced synaptic AMPAR incorporation (19).

The present study aimed to elucidate the effect of WM training on neurorehabilitation following focal cerebral ischemia. The ERK/CREB pathway and GluR2/GRASP-1/PICK1 cascades have been implicated in neuroplastic processes underlying recovery from ischemia. The current study further investigated the influence of WM training on these neural plasticity-associated signaling pathways.

\section{Materials and methods}

Animals and treatment regimen. Male Sprague Dawley rats ( $\mathrm{n}=160,200 \pm 20 \mathrm{~g}, 2$ months old) were obtained from the Central Animal House Facility of Xiangya Hospital (Changsha, China). The animal experiments were approved by the Animal Care Committee of Animal Ethics Committee of the Central South University. Animal care and all experimental procedures were conducted according to the guidelines of the care and use of laboratory animals (20). All animals were raised in plastic cages $\left(\mathrm{n}=4 /\right.$ cage, $\left.40 \times 30 \times 18 \mathrm{~cm}^{3}\right)$ with soft bedding and free access to food and water. Animals were housed at $23 \pm 2^{\circ} \mathrm{C}$ room temperature with a 12-h light/dark cycle.

Temporary middle cerebral artery occlusion (tMCAO) was performed according to a previously published protocol (21) with minor modifications. Prior to tMCAO surgery, animals were anesthetized deeply by an intraperitoneal injection of $2 \%$ pentobarbital sodium (30 mg/kg; Sigma-Aldrich; Merck KGaA, Darmstadt, Germany). A midline incision was made on the ventral surface of neck to expose the right common carotid artery. An intraluminal monofilament of filament (size 4.0, length $30 \mathrm{~mm}$ and diameter $0.19 \mathrm{~mm}$ ) with a silicon rubber coated tip was purchased from Beijing Cinontech Co., Ltd. (Beijing, China). It was inserted from the bifurcation of the common carotid artery into the internal carotid artery, and then into the beginning of the middle cerebral artery. Thread insertion was approximately $18.5 \pm 0.5 \mathrm{~mm}$ deep. Mild resistance indicated that the filament was properly lodged in the anterior cerebral artery and thus blocked blood flow to the middle cerebral artery. Reperfusion was obtained by withdrawing the filament gently after $2 \mathrm{~h}$.

Subsequent to $\mathrm{MCAO}$, the rats were randomly divided into 4 groups ( $\mathrm{n}=18$ rats/group): The tMCAO, control rehabilitation (CR), environment modification (EM) and willed movement (WM) groups, as presented in Fig. 1. In order to accustom the rats to the training regime, rats in the EM and WM groups were given 3 days of preliminary training for 10, 20 and $30 \mathrm{~min} /$ day on days 3,2 and 1 , respectively, prior to tMCAO surgery. Rats in the tMCAO group were raised in plastic cages 

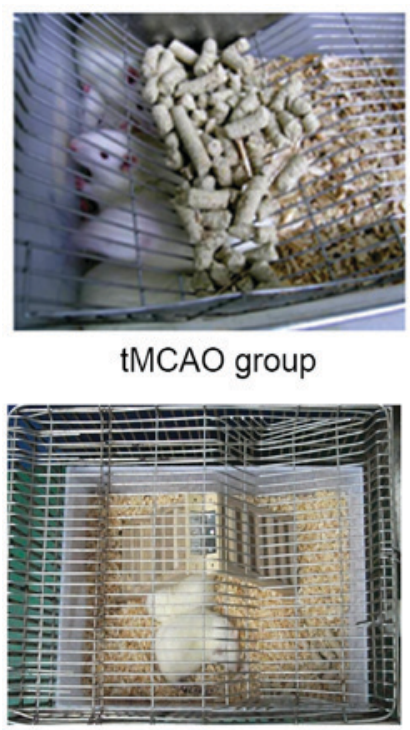

EM group
tMCAO group



CR group

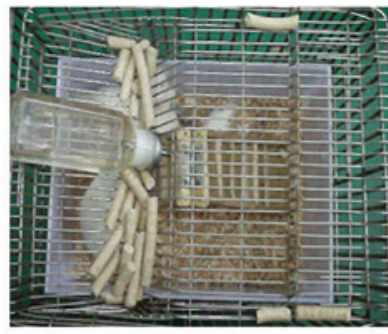

WM group
Figure 1. Neurorehabilitation exercises in each group. After the tMCAO, the rats were randomly divided into 4 groups ( $n=18$ rats in each group): The tMCAO group (no specific training), and the CR, EM and WM groups with different training methods. Rats in the CR group were forced to exercise (running) in a rotating wheel. In the WM group, food was used to entice rat to climb on a herringbone ladder. Herringbone ladders were also put into the cages of the CR and EM groups, however without the food attraction. tMCAO, temporary middle cerebral artery occlusion; CR, control rehabilitation; EM, environment modification; WM, willed movement.

without special rehabilitating training. Rats in the CR group were forced to exercise (running) in a rotating wheel $(100 \mathrm{~cm}$ long, $60 \mathrm{~cm}$ diameter) driven by hand. In the training, the running speed was set as 5 revolutions $/ \mathrm{min}$. Rats were forced to train twice a day respectively at 9:00 a.m. and 4:00 p.m. for 15 min per session. Following training, rats were returned to their cages and allowed free access to food and water.

A mini herringbone ladder was placed into the cages in the CR, EM and WM groups. Rats in the CR and EM groups could freely access food and water in cages independent of the herringbone ladder, however rats in the WM group had to climb the herringbone ladder to obtain food and water that were placed on the top of the cages. To ensure that both EM and WM rats consumed similar amounts of food and to ensure the attraction of the food for the WM rats, EM and WM rats were deprived of food for $12 \mathrm{~h}$ from 9:00 p.m. to 9:00 a.m. of the next day.

Neurobehavioral and neurological assessments: Frequency of rats climbing ladder. The behaviors of the rats climbing the herringbone ladders were observed twice a day at the following time periods: 9:00-10:00 a.m. and 3:00-4:00 p.m. on days $3,7,15$ and 30 post-surgery. The scoring method was: $1=$ climbing to the top of the herringbone ladder; $0.5=$ climbing half of the herringbone ladder. Investigators performing the outcome testing were kept blind to the groups.

Assessment of grip strength. The grip strength of rats was assessed using a string ( $\sim 50 \mathrm{~cm}$ in length) as described by Andrabi (22). The string was pulled tight between two vertical supports and elevated $40 \mathrm{~cm}$ from the flat surface. The rats were put on the string at the midway point and scoring was completed according to the following scoring scale: 0 , fall off; 1, hangs onto string by two forepaws; 2 , hangs on string by two forepaws and also attempts to climb on string; 3 , hangs onto string by two forepaws along with one or both hind paws; 4 , hangs onto string by all forepaws along with tail wrapped around the string; and 5, escape.

Neurological deficit scoring. Neurological deficit scoring is a combination of motor, sensory, reflex and balance tests. The neurological deficits were assessed on a four-point scale, according to previous studies $(23,24)$ with minor modifications. 0 points, no neurological damage, double forelimb symmetric stretching to the ground; 1 point, contralateral forelimb sustained adduction; 2 points, contralateral forelimb grip strength decreased; 3 points, slight stimulation of rat tails to the contralateral circling; 4 points, independent continuous circular motion. Motor functions were evaluated by three researchers who were kept blind to the groups. The animals in the model group with a score $\geq 1$ were selected for analysis in further experiments.

2,3,5-triphenyltetrazolium chloride (TTC) staining. After the completion of motor function evaluation, the animals were sacrificed and the brains were cut into $1.5 \sim 2 \mathrm{~mm}$ thick coronal sections. The brain slices were immersed in a $2 \%(\mathrm{w} / \mathrm{v})$ solution of TTC in normal saline at $37^{\circ} \mathrm{C}$ for $15 \mathrm{~min}$. TTC-stained brain slices were scanned and analyzed by a high-resolution scanner. The infarct volume was measured with Image J software, version 1.37 (National Institutes of Health, Bethesda, MD, USA).

Transmission election microscopy (TEM) observation. The ischemic brain tissues were dissected and post-fixed in $2.5 \%$ glutaraldehyde in phosphate-buffered saline (PBS) overnight. Subsequent to washing with PBS, the brain tissues were fixed in $0.1 \%$ osmium tetroxide for $2 \mathrm{~h}$, dehydrated in gradient ethanol and dimethyl ketone, and embedded in Epon 812 medium. The brain tissues were further dissected to ultrathin sections prior to observation using the transmission election microscope (Philips Tecnai-10; Phillips Electronic Instruments, Mahway, NJ, USA).

Immunohistochemistry and immunofluorescence. The cortical area and its adjacent corpus callosum in the ischemic hemisphere were isolated from brain and fixed with $4 \%$ paraformaldehyde for $20 \mathrm{~min}$ at room temperature. The slices were permeabilized with $0.3 \%$ Triton X-100 (Sigma-Aldrich; Merck $\mathrm{KGaA}$ ) for $10 \mathrm{~min}$ and subsequently blocked with $10 \%$ normal goat serum (Thermo Fisher Scientific, Inc., Waltham, MA, USA) in $0.01 \mathrm{M}$ PBS for $1 \mathrm{~h}$. Then, they were incubated overnight at $4^{\circ} \mathrm{C}$ with the primary antibodies against phosphorylated (p)-ERK (1:500 dilution; ab214036), pCREB (1:1,000 dilution; ab32096), GluR2 (1:800 dilution; ab133477), GRASP-1 (1:600 dilution; ab171940) and PICK1 (1:800 dilution; ab3420). These antibodies were purchased from Abcam (Cambridge, MA, USA). For immunohistochemistry, the slices were incubated with biotinylated horse-anti-rabbit IgG secondary antibody (1:100; OriGene Technologies, Inc., Beijing, China) for $1 \mathrm{~h}$, followed by incubation with avidin-biotin-peroxidase. For immunofluorescence, the slices were incubated with Alexa Fluor 594 anti-rabbit IgG secondary antibody (1:2,000; 
Table I. Rat mortality after tMCAO.

Death count after tMCAO

\begin{tabular}{lccccccc}
\cline { 2 - 6 } Groups & $0-24 \mathrm{~h}$ & $24-48 \mathrm{~h}$ & $48 \mathrm{~h}-3$ days & $3-7$ days & $7-15$ days & 15-30 days & Mortality (\%) \\
\hline MCAO & 8 & 8 & 2 & 4 & 2 & 1 & 53 \\
CR & 6 & 3 & 1 & 2 & 1 & 0 & 33 \\
EM & 7 & 5 & 2 & 3 & 2 & 1 & 45 \\
WM & 7 & 3 & 0 & 1 & 1 & 0 & 30 \\
\hline
\end{tabular}

MCAO, middle cerebral artery occlusion; $\mathrm{MCAO}$, temporary $\mathrm{MCAO}$; $\mathrm{CR}$, control rehabilitation; EM, environment modification; WM, willed movement.

Molecular Probes; Thermo Fisher Scientific, Inc.) and observed with the Nikon ECLIPSE Ti fluorescence microscope (Nikon Corporation, Tokyo, Japan) plus NIS-Elements BR 3.0 software (Nikon Corporation, Tokyo, Japan).

Western blotting. The cortex adjacent to the injury brain were isolated for protein harvest. These tissues were extracted with radioimmunoprecipitation assay lysis buffer (Beyotime Institute of Biotechnology, Shanghai, China) supplemented with phosphatase inhibitors. The concentration in the tissue samples was determined using the Bradford method (25), using a Bicinchoninic Acid Protein Assay kit (Beyotime Institute of Biotechnology). Protein lysates were separated by $10 \%$ SDS-PAGE and transferred to $0.45 \mu \mathrm{m}$ polyvinylidene difluoride membranes (EMD Millipore, Billerica, MA, USA). The membranes were blocked in $5 \%$ bovine serum albumin (Thermo Fisher Scientific, Inc.) overnight at $4^{\circ} \mathrm{C}$, followed by incubation in primary antibodies from Abcam against pERK (1:500 dilution; ab214036), CREB (1:800 dilution; ab32096), GluR2 (1:800 dilution; ab133477), GRASP-1 (1:900 dilution; ab171940), and PICK1 (1:800 dilution; ab3420) at $4^{\circ} \mathrm{C}$ overnight. Blots were washed $(3 \times 10 \mathrm{~min})$ in Tris-buffered saline containing 0.05\% Tween-20 (TBS-T; Sigma-Aldrich; Merck KGaA), following incubation with horseradish peroxidase-labeled donkey secondary antibody (cat. no. SA1-100, anti-mouse, 1:2,000; cat. no. SA1-200, anti-rabbit, 1:2,000; Invitrogen; Thermo Fisher Scientific, Inc.) and washed in TBS-T (3x10 min), protein bands were visualized using Amersham ECL Prime (GE Healthcare Life Sciences, Piscataway, NJ, USA) on the GelDoc XR System (Bio-Rad Laboratories, Inc., Hercules, CA, USA) and quantified by ImageJ software, version 9.0. The intensities of the protein bands were all normalized to $\beta$-actin.

Statistical analysis. All data were analyzed using one-way analysis of variance followed by Tukey's post hoc test. All data were analyzed using GraphPad Prism 5 software (GraphPad Software, Inc., La Jolla, CA, USA). P<0.05 was considered to indicate a statistically significant difference.

\section{Results}

Rat mortality after $t M C A O$. According to the data in Table I, rat mortality was higher during the first $24 \mathrm{~h}$ after tMCAO.
Table II. Frequency of rats climbing the herringbone ladders.

\begin{tabular}{lcccc}
\hline & \multicolumn{5}{c}{ Time after tMCAO (days) } \\
\cline { 2 - 5 } Groups & 3 & 7 & 15 & 30 \\
\hline EM & $3.22 \pm 0.79$ & $4.19 \pm 0.31$ & $4.68 \pm 0.34$ & $5.70 \pm 0.53$ \\
CR & $2.73 \pm 0.96$ & $4.06 \pm 0.14$ & $4.30 \pm 0.17$ & $5.58 \pm 0.25$ \\
WM & $3.47 \pm 0.84$ & $8.38 \pm 0.17^{\mathrm{a}, \mathrm{b}}$ & $10.72 \pm 0.37^{\mathrm{a}, \mathrm{b}}$ & $12.15 \pm 0.77^{\mathrm{a}, \mathrm{b}}$
\end{tabular}

${ }^{\mathrm{a}} \mathrm{P}<0.05$, vs. EM group; ${ }^{\mathrm{b}} \mathrm{P}<0.05, \mathrm{WM}$ group vs. $\mathrm{CR}$ group $(\mathrm{n}=12)$. tMCAO, temporary middle cerebral artery occlusion; EM, environment modification; CR, control rehabilitation; WM, willed movement.

Table III. Grip strength of rats.

\begin{tabular}{lcccc}
\hline & \multicolumn{5}{c}{ Time after tMCAO (days) } \\
\cline { 2 - 5 } Groups & 3 & 7 & 15 & 30 \\
\hline EM & $0.73 \pm 0.47$ & $0.91 \pm 0.30$ & $1.33 \pm 0.49$ & $2.52 \pm 0.46$ \\
CR & $0.55 \pm 0.52$ & $0.83 \pm 0.39$ & $1.25 \pm 0.45$ & $2.46 \pm 0.38$ \\
WM & $0.67 \pm 0.49$ & $0.91 \pm 0.29$ & $1.75 \pm 0.48^{\mathrm{a}, \mathrm{b}}$ & $2.92 \pm 0.29^{\mathrm{a}, \mathrm{b}}$ \\
\hline
\end{tabular}

${ }^{\mathrm{a}} \mathrm{P}<0.05$, vs. EM group; ${ }^{\mathrm{b}} \mathrm{P}<0.05, \mathrm{WM}$ group vs. $\mathrm{CR}$ group $(\mathrm{n}=12)$. tMCAO, temporary middle cerebral artery occlusion; EM, environment modification; $\mathrm{CR}$, control rehabilitation; WM, willed movement.

In the second $24 \mathrm{~h}$, rat mortality was decreased in the CR, EM and WM groups, however not in the tMCAO group. Rat mortality in all groups was progressively reduced during the period of $48 \mathrm{~h} \sim 30$ days after tMCAO. The WM group exhibited the lowest mortality in the first month after tMCAO among these groups.

Neurobehavioral analysis. In order to analyze the effect of WM training on attenuation of the neurological deficits after tMCAO surgery, several behavioral parameters that could to reflect motor ability and coordination were studied. The number of times that rats climbed the ladders was recorded at indicated times after 
Table IV. Neurological deficit scores.

Time after tMCAO

\begin{tabular}{lcccccc}
\cline { 2 - 6 } Groups & $1 \mathrm{~h}$ & $24 \mathrm{~h}$ & 3 days & 7 days & 15 days & 30 days \\
\hline tMCAO & $3.08 \pm 0.29$ & $2.08 \pm 0.29$ & $1.92 \pm 0.29$ & $1.75 \pm 0.45$ & $1.50 \pm 0.52$ & $1.33 \pm 0.49$ \\
EM & $3.00 \pm 0.60$ & $2.17 \pm 0.39$ & $1.83 \pm 0.39$ & $1.58 \pm 0.51$ & $1.33 \pm 0.49$ & $1.08 \pm 0.79$ \\
CR & $3.08 \pm 0.29$ & $2.08 \pm 0.29$ & $1.83 \pm 0.39$ & $1.67 \pm 0.49$ & $1.41 \pm 0.51$ & $1.17 \pm 0.72$ \\
WM & $3.08 \pm 0.40$ & $2.17 \pm 0.39$ & $1.75 \pm 0.45$ & $1.50 \pm 0.52$ & $1.25 \pm 0.45$ & $0.75 \pm 0.62^{\text {a-c }}$ \\
\hline
\end{tabular}

${ }^{\mathrm{a}} \mathrm{P}<0.05$, other groups vs. tMCAO group; ${ }^{\mathrm{P}} \mathrm{P}<0.05$, WM and $\mathrm{CR}$ groups vs. EM group; ${ }^{\mathrm{c}} \mathrm{P}<0.05$, WM group vs. CR group (n=12). tMCAO, temporary middle cerebral artery occlusion; EM, environment modification; CR, control rehabilitation; WM, willed movement.

surgery. As presented in Table II, the climbing frequencies of rats in the WM group were notably increased 7 days following surgery. In contrast, the climbing frequencies of rats in the CR and EM groups were increased moderately 30 days after surgery. The climbing frequencies of rats were significantly higher in the WM group compared with that of the CR and EM groups on days 7, 15 and 30 subsequent to surgery $(\mathrm{P}<0.05)$.

The grip strength of rats in the CR, EM and WM groups was observed subsequent to surgery. The mean reading of three successive trials for each rat was taken as a dependent variable. The scoring reflected that there was a gradual increase in the grip strength of rats in these groups 30 days after surgery. Notably, the grip strength of WM rats was higher compared with CR and EM rats on days 15 and 30 after surgery $(\mathrm{P}<0.05$; Table III).

The neurological deficit scores did not differ significantly among the four groups within 15 days after recirculation. However, neurological deficit scores were significantly reduced in the WM group compared with other groups 30 days after surgery $(\mathrm{P}<0.05$; Table IV).

Infarct volume. TTC staining is a classical method to assess area of cerebral infarctions caused by MCAO. Normal cerebral tissue was stained red, and unstained areas were defined as the infarcted tissue. As presented in Fig. 2 and Table V, all groups exhibited a gradual reduction in the infarct volume percentage during the first month after the tMCAO. Infarct volume percentage was notably decreased in the WM group on days $15(\mathrm{P}<0.05$ vs. tMCAO group) and $30(\mathrm{P}<0.05$ vs. other groups) in addition to in the $\mathrm{CR}$ group on days 7 and 15 ( $\mathrm{P}<0.05$ vs. $\mathrm{tMCAO}$ group).

TEM observation. TEM observation indicated the ultrastructural changes of nerve cells after stroke (Fig. 3). A total of 3 days after $\mathrm{tMCAO}$, there were few synaptic junctions present in each group. WM and CR groups exhibited notable increases in the number of synaptic junctions 30 days after stroke, however the increase was moderate in the EM and tMCAO groups.

Location and expression of indicated protein in ischemic penumbra of brain. Immunohistochemical staining demonstrated the location and expression levels of GluR2 and GRASP-1 in the neurons. GluR2 and GRASP-1 were
Table V. Infarct volume indicated by 2,3,5-triphenyltetrazolium chloride staining.

\begin{tabular}{lcccc}
\hline & \multicolumn{5}{c}{ Time after tMCAO (days) } \\
\cline { 2 - 5 } Groups & 3 & 7 & 15 & 30 \\
\hline MCAO & $0.20 \pm 0.10$ & $0.18 \pm 0.19$ & $0.15 \pm 0.18$ & $0.08 \pm 0.14$ \\
EM & $0.18 \pm 0.25$ & $0.17 \pm 0.13$ & $0.14 \pm 0.18$ & $0.08 \pm 0.25$ \\
CR & $0.19 \pm 0.15$ & $0.15 \pm 0.14^{\mathrm{a}}$ & $0.11 \pm 0.14^{\mathrm{a}}$ & $0.07 \pm 0.21$ \\
WM & $0.17 \pm 0.19$ & $0.16 \pm 0.08$ & $0.12 \pm 0.16^{\mathrm{a}}$ & $0.03 \pm 0.78^{\mathrm{a}-\mathrm{c}}$
\end{tabular}

${ }^{\mathrm{a}} \mathrm{P}<0.05$, other groups vs. tMCAO group; ${ }^{\mathrm{b}} \mathrm{P}<0.05$, WM and $\mathrm{CR}$ groups vs. EM group; ${ }^{\mathrm{P}}<0.05$, WM group vs. $\mathrm{CR}$ group $(\mathrm{n}=12)$. tMCAO, temporary middle cerebral artery occlusion; EM, environment modification; CR, control rehabilitation; WM, willed movement.

expressed in the cytoplasm and the cell nucleus. The proportion of GluR2-stained neurons was higher in CR (47\%), EM $(53 \%)$ and WM (82\%) groups compared with that of the tMCAO (38\%) group. There were no significant differences in GRASP-1-stained cells among tMCAO, CR and EM groups. The WM group exhibited increased GRASP-1 staining compared with the other groups (Fig. 4A).

Neurons with pERK and pCREB staining presented with green fluorescence. pERK staining was present in the cytoplasm and cell nucleus of neurons, while pCREB staining was present in the cell nucleus. The WM group exhibited an increase in pERK-stained neurons compared with other groups. The proportion of pCREB-stained neurons was $\sim 28,75,67$ and $83 \%$ in the tMCAO, CR, EM and WM groups, respectively (Fig. 4B).

GluR2 and PICK1 expression was detected with immunofluorescence. Neurons with GluR2 and PICK1 staining were observed to be increased in the WM group compared with the tMCAO group (Fig. 4C).

Western blotting was conducted in order to quantify expression of these proteins. pERK and pCREB protein levels were increased in $\mathrm{CR}(\mathrm{P}<0.01), \mathrm{EM}(\mathrm{P}<0.05)$, and WM $(\mathrm{P}<0.001)$ groups compared with the tMCAO group. In addition, expression levels of GluR2, GRASP-1 and PICK1 were increased in CR $(\mathrm{P}<0.05)$, EM $(\mathrm{P}<0.05)$ and WM $(\mathrm{P}<0.01)$ groups (Fig. 5). 

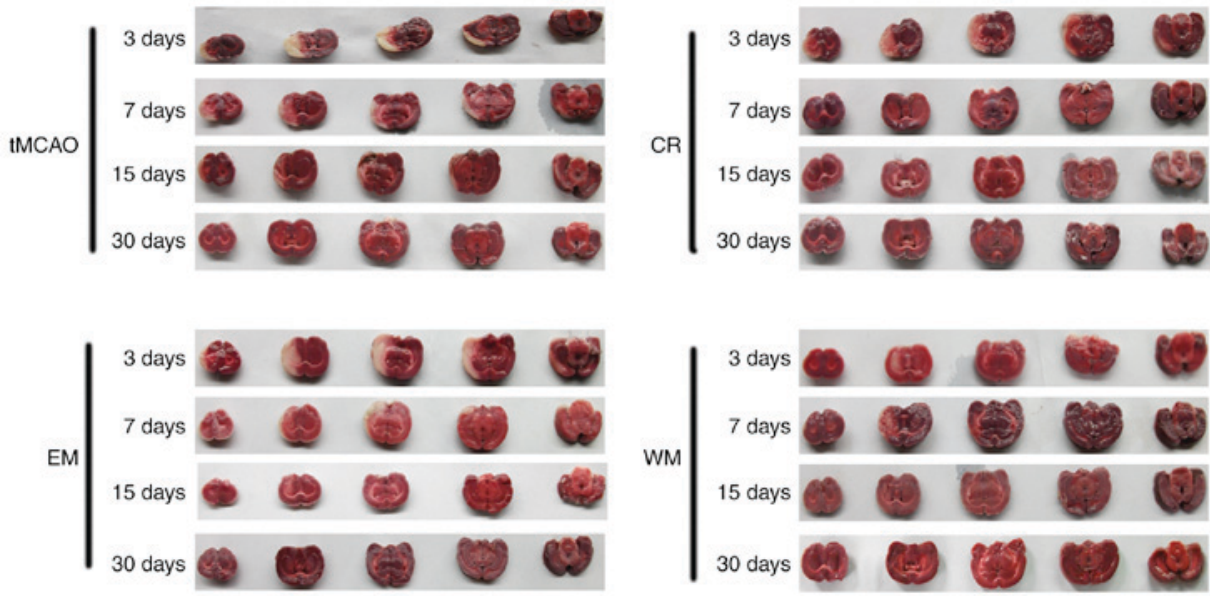

Figure 2. TTC staining in each group. After the tMCAO, the rats were randomly divided into 4 groups (n=18 rats in each group): The tMCAO group (no specific training), and the CR, EM and WM groups with different training methods. The rats in each groups were sacrificed and the brains were stained with TTC TTC, 2,3,5-triphenyltetrazolium chloride; tMCAO, temporary middle cerebral artery occlusion; CR, control rehabilitation; EM, environment modification; WM, willed movement.

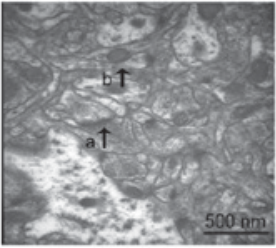

Normal

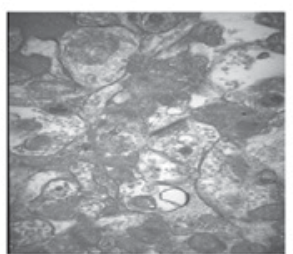

3 days

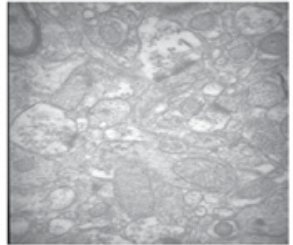

30 days

IMCAO



3 days



30 days

EM



3 days

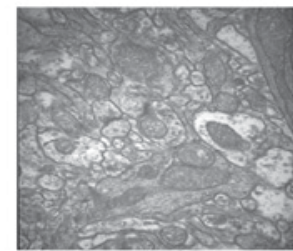

3 days

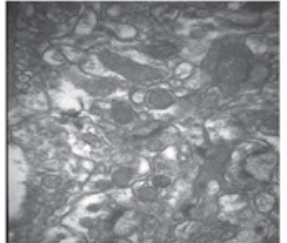

30 days

CR

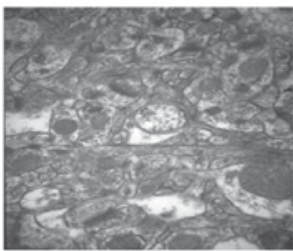

30 days

WM

Figure 3. TEM observation. After the tMCAO, the rats were randomly divided into 4 groups ( $\mathrm{n}=18$ rats in each group): The tMCAO group (no specific training), and the CR, EM and WM groups with different training methods. The brain of the injured side was evaluated with TEM observation. a indicates normal neurons, b indicates synaptic junctions. TEM, transmission electron microscopy; tMCAO, temporary middle cerebral artery occlusion; CR, control rehabilitation; EM, environment modification; WM, willed movement.

\section{Discussion}

Post-stroke neurological injury is an important health problem associated with a high disability and mortality rate worldwide (1). Stroke is associated with cerebrovascular bleeding, inflammatory reaction and stress response, which causes neuronal apoptosis or death and functional loss of motion, communication and cognition (2). Neurorehabilitation training represents a therapeutic option to help stroke patients improve the impaired nervous system and motor control, according to the activity-dependent neural plasticity. Exercise training has been considered to improve neuroplasticity, which leads to enhancement, compensation and replacement of the remaining function of nerve (24). Synaptic activity is decreased following brain injury, however exercise training increases presynaptic release of neurotransmitters, in addition to upregulating postsynaptic response to those neurotransmitters, which can, at least in part, restore neural function following an injury (26). In addition to synaptic activity regulation, new synapse formation, which may compensate for the lost structural circuits, can be triggered by stroke. This self-regulation of synaptic plasticity is activity- and experience-dependent (27). Motion-rehabilitation training can increase the connectivity between multiple brain regions that are disconnected after stroke, leading to improved functional outcomes.

In the present study, rats in the WM group exhibited greater improvements in neurobehavioral performance compared with the other groups. The WM group exhibited an evident reduction in brain damage as indicated by TTC staining. TEM observation indicated that there were increased synaptic junctions following WM training for a period of time (one month). A previous study demonstrated that WM training was more effective in enhancing the PICK1-mediated synaptic plasticity in the area adjacent to the damage region of ischemic rats 
A
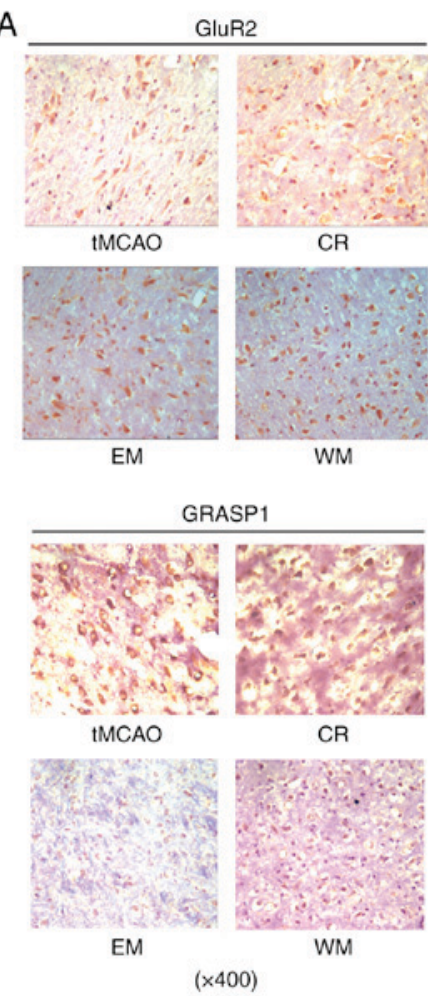

B


tMCAO
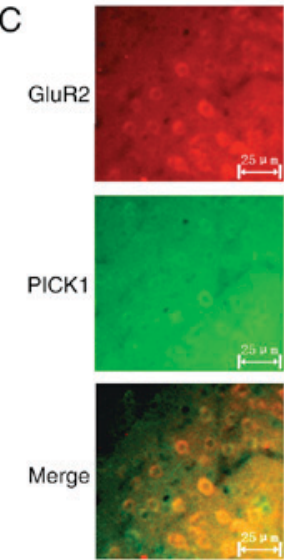
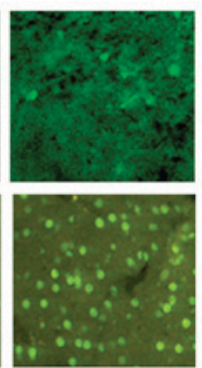

CR
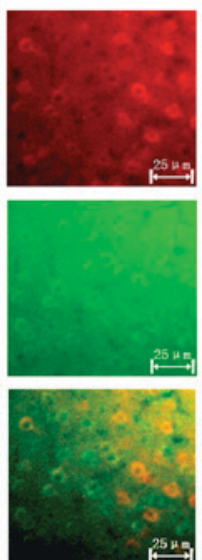

CR
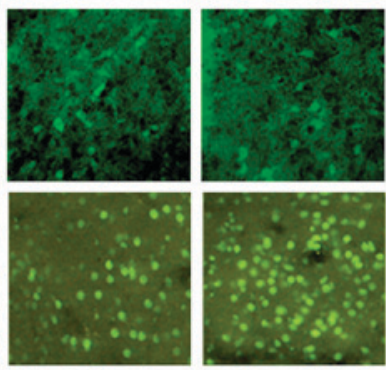

EM

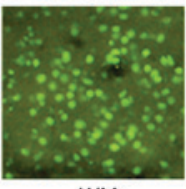

WM
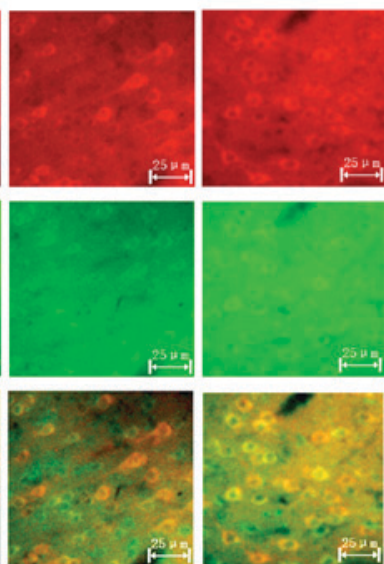

EM

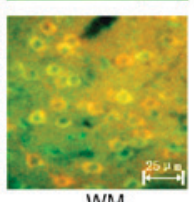

Figure 4. Immunohistochemistry and immunofluorescence. After the tMCAO, the rats were randomly divided into 4 groups (n=18 rats in each group): The tMCAO group (no specific training), and the CR, EM and WM groups with different training methods. The cortical area and its adjacent corpus callosum in the ischemic hemisphere were evaluated with (A) immunohistochemistry and (B and C) immunofluorescence methods to detect the expression of pERK, pCREB, GluR2, GRASP-1 and PICK1. tMCAO, temporary middle cerebral artery occlusion; CR, control rehabilitation; EM, environment modification; WM, willed movement; p-, phosphorylated; ERK, extracellular signal-related kinase; CREB, cyclic adenosine monophosphate response element-binding protein 1; GluR2, glutamate receptor 2; GRASP-1, glutamate receptor interacting protein 1-associated protein 1; PICK1, protein interacting with C-kinase 1.
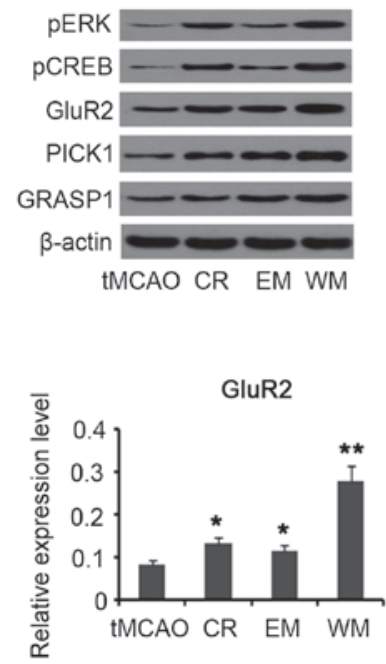

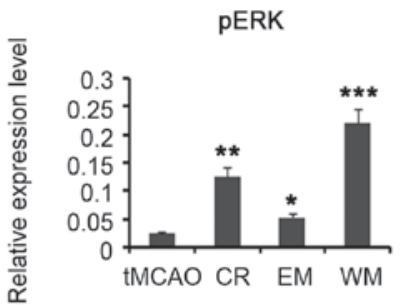

PICK1

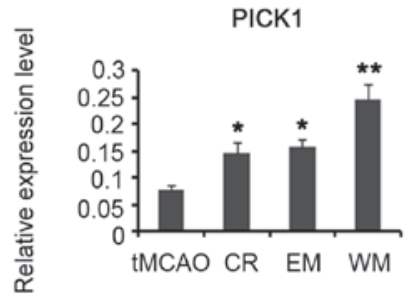

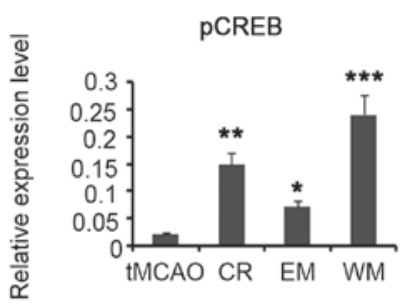

GRASP1

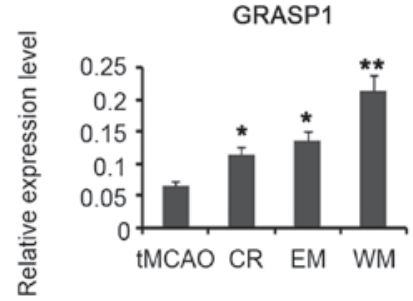

Figure 5. Quantification of protein level with western blotting. Subsequent to tMCAO, the rats were randomly divided into 4 groups ( $\mathrm{n}=18 \mathrm{rats}$ in each group): The tMCAO group (no specific training), and the CR, EM and WM groups with different training methods. Expression of pERK, pCREB, GluR2, GRASP-1 and PICK1 levels in the cortex adjacent to the injury brain were evaluated with western blotting. ${ }^{*} \mathrm{P}<0.05$ and ${ }^{* *} \mathrm{P}<0.01$ vs. tMCAO group. tMCAO, temporary middle cerebral artery occlusion; CR, control rehabilitation; EM, environment modification; WM, willed movement; p-, phosphorylated; ERK, extracellular signal-related kinase; CREB, cyclic adenosine monophosphate response element-binding protein 1; GluR2, glutamate receptor 2; GRASP-1, glutamate receptor interacting protein 1-associated protein 1; PICK1, protein interacting with C-kinase 1.

when compared with swimming training (28). Traditional neurorehabilitation training highlights the intensity, however fails to take into account the voluntary participation of patients. Ploughman et al (29) observed that although the more intense, motorized running exercise induced a rapid increase in BDNF, the elevation was more short-lived compared to that 
of voluntary running. In addition, intense motorized running triggered a more pronounced increase in the stress hormone, corticosterone, however reduction in pCREB and synapsin-I in hippocampus were observed beginning 30 to $60 \mathrm{~min}$ subsequent to the exercise (29). It is, thus, suggested that frequent voluntary running with a lower intensity should be used in the clinic, which may have a delayed but sustained effect supporting brain remodeling after stroke.

In a previous study, it was identified that the signal transducer and activator of transcription 3 (STAT3) pathway was involved in the regulation of BDNF, PICK1 and SYP and mediates the improvements in neuroplasticity that result from WM training (30). The present study additionally identified that the ERK/CREB pathway was notably activated following WM training. Although CR and EM groups also exhibited ERK/CREB pathway activation, this was not as effective as that of the WM group. Notably, BDNF and SYP have been observed to be positively regulated by the ERK pathway $(8,9)$, it is suggested that STAT3 and ERK signaling may exert synergistic or additional effects on the promotion of BDNF and SYP. The ERK/CREB pathway serves as a key signaling pathway for neuroprotection and endogenous neurogenesis. It was reported that CREB activation serves a critical part in DNA repair and anti-oxidation, thereby protecting neurons from damage induced by cerebral ischemia and reperfusion (9). Neurogenesis mediated by the ERK/CREB pathway is involved in structural and functional neuroplasticity, therefore, it is suggested that ERK/CREB activation triggered by WM training is important to reduce brain damage after stroke, and improves neurobehavioral performance.

It has been previously observed that AMPAR is involved in neuroplasticity improved by WM training (21). In the mammalian central nervous system, AMPA-type glutamate receptors mediate the majority of fast excitatory synaptic transmission. AMPARs are tetramers made up of combinations of four subunits: GluR1, GluR2, GluR3 and GluR4, andGluR1 and GluR4 mRNA of rats in the WM group have been observed to be significantly upregulated in the ischemia penumbra region at the subacute stage (21). The present study used western blotting, immunofluorescence and immunohistochemistry to indicate that GluR2 was also upregulated following WM training. GluR2 is a critical subunit in determining mammalian AMPAR function; GluR2 is involved in the regulation of the $\mathrm{Ca}^{2+}$ permeability, single-channel conductance and AMPAR trafficking and assembling. AMPAR trafficking, namely redistribution in and out of the synapse, has emerged as an important mechanism for synaptic plasticity, such as LTP and long-term depression (LTD) (16). Increased delivery of AMPARs to the postsynaptic membrane leads to LTP, while net removal of AMPARs by internalization from the surface appears to underlie LTD (17). GRASP-1 affects GluR2/3-GRIPinteraction and regulates AMPAR trafficking to the synaptic membrane, thus it is associated to AMPAR-dependent LTP (14). PICK1 is an AMPAR-binding protein, serving an essential role in regulating LTD in cerebral and hippocampal synapses. A previous study identified that WM training induces a PICK1-dependent LTD in rats subjected to focal cerebral ischemia (30). In the present study, it was observed that
GRASP-1 and PICK1 expression were induced by WM training, suggesting that WM training is involved in LTP and LTD in regulating synaptic plasticity.

Collectively, the present study demonstrated that WM training confers greater effects on improving neurobehavioral performance compared with traditional training in a rotating wheel. The WM group exhibited evident reduction in brain damage with increased synaptic junctions. Study of the molecular mechanism indicated that WM training promotes the ERK/CREB pathway and GluR2/GRASP-1/PICK1 cascades. These signaling pathways were involved in neuroprotection, endogenous neurogenesis and synaptic plasticity of LTP and LTD. The current study lays a preliminary foundation for future research on therapeutic intervention of WM training against stroke-induced neuronal damage.

\section{Acknowledgements}

The present study was supported by grants from the National Natural Science Foundation of China (grant no. 30973167), China Postdoctoral Science Foundation (grant no. 2011m501301), Postdoctoral Science Foundation of Hunan Province and the Postdoctoral Science Foundation of Central South University.

\section{References}

1. Pan L, Song A, Duan S and Yu Z: Patient-centered robot-aided passive neurorehabilitation exercise based on safety-motion decision-making mechanism. Biomed Res Int 2017: 4185939, 2017.

2. Chen X, Zhang X, Xue L, Hao C, Liao W and Wan Q: Treatment with enriched environment reduces neuronal apoptosis in the periinfarct cortex after cerebral ischemia/reperfusion injury. Cell Physiol Biochem 41: 1445-1456, 2017.

3. Parker K, Berretta A, Saenger S, Sivaramakrishnan M, Shirley SA, Metzger F and Clarkson AN: PEGylated insulin-like growth factor-I affords protection and facilitates recovery of lost functions post-focal ischemia. Sci Rep 7: 241, 2017.

4. Choi DH, Ahn JH, Choi IA, Kim JH, Kim BR and Lee J: Effect of task-specific training on Eph/ephrin expression after stroke. BMB Rep 49: 635-640, 2016.

5. Sanchez-Mendoza EH and Hermann DM: Correlates of post-stroke brain plasticity, relationship to pathophysiological settings and implications for human proof-of-concept studies. Front Cell Neurosci 10: 196, 2016.

6. Tang QP, Shen Q, Wu LX, Feng XL, Liu H, Wu B, Huang XS, Wang GQ, Li ZH and Liu ZJ: STAT3 signal that mediates the neural plasticity is involved in willed-movement training in focal ischemic rats. J Zhejiang Univ Sci B 17: 493-502, 2016.

7. Ye Y, Zhao Z, Xu H, Zhang X, Su X, Yang Y, Yu X and He X: Activation of sphingosine 1-phosphate receptor 1 enhances hippocampus neurogenesis in a rat model of traumatic brain injury: An involvement of MEK/Erk signaling pathway. Neural Plast 2016: 8072156, 2016.

8. Liu L, Zhu J, Zhou L and Wan L: RACK1 promotes maintenance of morphine-associated memory via activation of an ERK-CREB dependent pathway in hippocampus. Sci Rep 6: 20183, 2016.

9. Li Y, Li X, Guo C, Li L, Wang Y, Zhang Y, Chen Y, Liu W and Gao L: Long-term neurocognitive dysfunction in offspring via NGF/ERK/CREB signaling pathway caused by ketamine exposure during the second trimester of pregnancy in rats. Oncotarget 8: 30956-30970, 2017.

10. Qu H, Zhao M, Zhao S, Xiao T, Tang X, Zhao D, Jolkkonen J and Zhao C: Forced limb-use enhances brain plasticity through the cAMP/PKA/CREB signal transduction pathway after stroke in adult rats. Restor Neurol Neurosci 32: 597-609, 2014. 
11. Li H, Wang J, Wang P, Rao Y and Chen L: Resveratrol reverses the synaptic plasticity deficits in a chronic cerebral hypoperfusion rat model. J Stroke Cerebrovasc Dis 25: 122-128, 2016.

12. Pregi N, Belluscio LM, Berardino BG, Castillo DS and Cánepa ET: Oxidative stress-induced CREB upregulation promotes DNA damage repair prior to neuronal cell death protection. Mol Cell Biochem 425: 9-24, 2017.

13. Isaac JT, Ashby MC and McBain CJ: The role of the GluR2 subunit in AMPA receptor function and synaptic plasticity. Neuron 54: 859-871, 2007.

14. Dixon RM, Mellor JR and Hanley JG: PICK1-mediated glutamate receptor subunit 2 (GluR2) trafficking contributes to cell death in oxygen/glucose-deprived hippocampal neurons. J Biol Chem 284: 14230-14235, 2009.

15. Hanley JG: Actin-dependent mechanisms in AMPA receptor trafficking. Front Cell Neurosci 8: 381, 2014.

16. Bakshi K, Kosciuk M, Nagele RG, Friedman E and Wang HY: Prenatal cocaine exposure increases synaptic localization of a neuronal RasGEF, GRASP-1 via hyperphosphorylation of AMPAR anchoring protein, GRIP. PLoS One 6 : e25019, 2011

17. Hoogenraad CC and van der Sluijs P: GRASP-1 regulates endocytic receptor recycling and synaptic plasticity. Commun Integr Biol 3: 433-435, 2010.

18. Ye B, Yu WP, Thomas GM and Huganir RL: GRASP-1 is a neuronal scaffold protein for the JNK signaling pathway. FEBS Lett 581: 4403-4410, 2007.

19. Chiu SL, Diering GH, Ye B, Takamiya K, Chen CM, Jiang Y, Niranjan T, Schwartz CE, Wang T and Huganir RL: GRASP1 regulates synaptic plasticity and learning through endosomal recycling of AMPA receptors. Neuron 93: 1405-1419. e8, 2017.

20. Institute for Laboratory Animal Research. Guide for the care and use of laboratory animals, 8th edition Washington (DC): National Academies Press, 2011

21. Tang Q, Yang Q, Hu Z, Liu B, Shuai J, Wang G, Liu Z, $X i a J$ and Shen $X$ : The effects of willed movement therapy on AMPA receptor properties for adult rat following focal cerebral ischemia. Behav Brain Res 181: 254-261, 2007.
22. Andrabi SS, Parvez S and Tabassum H: Progesterone induces neuroprotection following reperfusion-promoted mitochondrial dysfunction after focal cerebral ischemia in rats. Dis Model Mech 10: 787-796, 2017.

23. Shu S, Li CM, You YL, Qian XL, Zhou S and Ling CQ: Electroacupuncture ameliorates cerebral ischemia-reperfusion injury by regulation of autophagy and apoptosis. Evid Based Complement Alternat Med 2016: 7297425, 2016.

24. Chen L, Wang X, Zhang J, Dang C, Liu G, Liang Z, Huang G, Zhao $\mathrm{W}$ and Zeng J: Tongxinluo enhances neurogenesis and angiogenesis in peri-infarct area and subventricular zone and promotes functional recovery after focal cerebral ischemic infarction in hypertensive rats. Evid Based Complement Alternat Med 2016: 8549590, 2016.

25. Cheng Y, Wei H, Sun R, Tian Z and Zheng X: Rapid method for protein quantitation by Bradford assay after elimination of the interference of polysorbate 80 . Anal Biochem 494: 37-39, 2016.

26. Fu J, Wang H, Deng L and Li J: Exercise training promotes functional recovery after spinal cord injury. Neural Plast 2016: 4039580, 2016

27. Cheng A, Hou Y and Mattson MP: Mitochondria and neuroplasticity. ASN Neuro 2: e00045, 2010.

28. Nie J, Yang X, Tang Q, Shen Q and Li S: Willed-movement training reduces brain damage and enhances synaptic plasticity related proteins synthesis after focal ischemia. Brain Res Bull 120: 90-96, 2016

29. Ploughman M, Granter-Button S, Chernenko G, Attwood Z, Tucker BA, Mearow KM and Corbett D: Exercise intensity influences the temporal profile of growth factors involved in neuronal plasticity following focal ischemia. Brain Res 1150: 207-216, 2007.

30. Tang Q, Tan L, Yang X, Shen Q, Huang X, Wang G, Chen H, Nie J, Li S and Wu L: Willed-movement training reduces motor deficits and induces a PICK1-dependent LTD in rats subjected to focal cerebral ischemia. Behav Brain Res 256: 481-487, 2013 